\title{
Simulation of Coherent Radar Imaging Using Continuous Wave Noise Radar
}

\author{
JING XU AND M. W. HOFFMAN \\ Department of Electrical Engineering, University of Nebraska at Lincoln, Lincoln, Nebraska \\ B. L. CHEONG AND R. D. PALMER \\ School of Meteorology, and Atmospheric Radar Research Center, University of Oklahoma, Norman, Oklahoma
}

(Manuscript received 26 June 2008, in final form 10 January 2009)

\begin{abstract}
A computationally simple cross-correlation model for multiple backscattering from a continuous wave (CW) noise radar is developed and verified with theoretical analysis and brute-force time-domain simulations. Based on this cross-correlation model, a modification of an existing numerical method originally developed by Holdsworth and Reid for spaced antenna (SA) pulsed radar is used to simulate the estimated cross correlation corresponding to atmospheric backscattering using a coherent CW noise radar. Subsequently, coherent radar imaging (CRI) processing comparisons between the $\mathrm{CW}$ noise radar and a conventional pulsed radar are presented that verify the potential of $\mathrm{CW}$ noise radar for atmospheric imaging.
\end{abstract}

\section{Introduction}

By applying radar to atmospheric remote sensing, atmospheric parameters can be derived after processing the received signals. Return power variations and Doppler shifts are caused by fluctuations in the atmospheric refractive index that are in turn affected by humidity, pressure, temperature, and mass density (Doviak and Zrnić 1993). Atmospheric remote sensing using radar has been extensively studied for many years by using conventional pulsed radars and Doppler radar. Recently, increasing interest has been seen for atmospheric applications using passive noise radar (Sahr and Lind 1997; Meyer and Sahr 2004; Sahr and Meyer 2004), for simulation of land and rain clutter at the $\mathrm{X}$ band using pseudorandom code (PRC) continuous wave $(\mathrm{CW})$ radar (Zhang et al. 1999), and for the consideration of noise radar in weather applications (Yanovsky 2002).

Coherent radar imaging (CRI), also referred to as beam forming in many fields, is based on sensor array signal processing techniques. CRI allows observations of small-scale structure in reflectivity maps and wind fields and its application has become more common in

Corresponding author address: Michael W. Hoffman, Department of Electrical Engineering, University of Nebraska at Lincoln, Lincoln, NE 68588-0511.

E-mail:mhoffman1@unl.edu the atmospheric remote sensing area. CRI improvements have included increased angular resolution and enhanced robustness. Applications of CRI techniques (Kudeki and Sürücü 1991; Hysell 1996) can be found in studies of the mesosphere (Yu et al. 2001) and the atmospheric boundary layer (ABL; Mead et al. 1998; Pollard et al. 2000; Cheong et al. 2004b).

A CW noise radar transmits and receives random noise or noiselike waveforms for target illumination. Early development of this basic technique includes the work of Waltman et al. (1966), Reid (1969), and Krehbiel and Brook (1979). In Waltman et al. (1966) a broadband two-element interferometer is described that uses a random noise waveform. In Reid (1969) drop size spectral analysis is performed using a pseudorandom phase code-modulated radar. In Krehbiel and Brook (1979) a broadband noise radar that reduces the between scatterer interference within a given volume is described. This approach reduces required averaging and allows for faster scanning. Because of the random nature of the transmitted signal, noise radar has advantages of low probability of intercept (LPI), good accuracy and resolution, unambiguous measurement of distance and velocity, and counter electronic support measure capability (Guosui et al. 1999). Given these advantages, random noise radar has been used in a wide range of applications including surveillance, tracking, collision warning, and air defense. As can be seen from 
the cited work, these advantages are also attractive for atmospheric remote sensing.

The present work attempts to verify a simple computational model for CRI simulations of atmospheric observations using a coherent $\mathrm{CW}$ noise radar. A CW correlation model for a single scatterer is developed with notation that is compatible with existing CRI simulation approaches. This model is first verified through simulation comparisons of the simple computational model obtained via theoretical analysis and a computationally intense time-domain simulation. Subsequently, an application of this simplified model to atmospheric CRI is done via simulation in parallel with the previously established pulsed radar CRI techniques. A primary motivation of this work is to enable development of passive atmospheric imaging radars that exploit existing broadband communications signals. This passive radar development is similar to the work in Sahr and Lind (1997), but is intended for use in radar imaging of the lower atmosphere. This computationally simple tool provides developers of passive atmospheric imaging radar systems the ability to compare $\mathrm{CW}$ approaches with existing pulsed radars such as TEP (see, e.g., Cheong et al. 2004b). In particular, this tool allows the analysis of the basic parameter trades including duty cycle, SNR averaging, and transmit-receive cross-talk reduction.

\section{Overview of coherent $\mathrm{CW}$ noise radar}

\section{a. Basic principles of $C W$ noise radar}

In a CW noise radar both range and radial velocity estimation are accomplished by processing the cross correlation of the received signal and a delayed version of the transmitted signal. The cross correlation for a point scatterer is given by

$$
R_{r d}(t)=E\left[x_{r}(t) x_{d}(t)\right]=A R_{x}(t, \tau),
$$

where $E[\cdot]$ denotes the expected value operator; $x_{r}(t)$ represents the received signal from a point scatterer, which is a time and/or Doppler shifted version of the transmitted signal; $x_{d}(t)$ represents the delayed replica of the transmitted signal; $A$ is the amplitude scaling factor; $R_{x}(t, \tau)$ is the autocorrelation of the transmitted signal; and $\tau$ is the difference of the return delay $\tau_{r}$ and the delay of the replica $\tau_{d}$ (i.e., $\tau=\tau_{r}-\tau_{d}$ ).

For a band-limited stationary random process with uniform (i.e., flat) power spectral density (PSD) centered at the frequency $f_{0}$, its autocorrelation $R_{x}(\tau)$ is a $\operatorname{sinc}()$ function modulated by a sinusoidal function with center frequency $f_{0}$ (Dawood 2001). Therefore, for a $\mathrm{CW}$ noise radar transmitting bandpass random noise with a uniform PSD, the cross correlation given by Eq. (1) can be described by

$$
R_{r d}(t, \tau)=A \frac{\sin (\pi \beta \tau)}{\pi \beta \tau} \cos \left(2 \pi f_{0} \tau\right)
$$

where $f_{0}$ is the carrier frequency (in $\mathrm{Hz}$ ) and $\beta$ represents the transmit bandwidth (in $\mathrm{Hz}$ ). A strong correlation peak occurs when the delayed replica matches the return signal in delay time (i.e., $\tau_{d}=\tau_{r}$ ), so the range detection is based on estimating $\tau_{d}$ corresponding the crosscorrelation peak. For a moving point scatterer, $\tau$ is a function of $t$ and its velocity can be estimated by finding the center frequency of the correlation time series.

\section{b. Outputs of coherent $C W$ noise radar}

In a practical $\mathrm{CW}$ noise radar, assuming the transmitted noise wave is a wide sense stationary (WSS) ergodic random process, the cross-correlation of the received signal and a delayed replica can be approximated in the time-averaged sense (Dawood 2001):

$$
\hat{R}_{r d}(t, \tau) \frac{1}{T_{\mathrm{int}}} \int_{t}^{t+T_{\mathrm{int}}} x_{r}(\alpha) x_{d}(\alpha) d \alpha,
$$

where $T_{\text {int }}$ is the integration time. In a coherent $\mathrm{CW}$ noise radar system (Narayanan et al. 1998), $x_{d}(t)$ can be a time delayed and frequency shifted (by $f_{\mathrm{IF}}$ ) replica of the transmitted signal, the product $x_{r}(t) x_{d}(t)$ is passed through a bandpass filter with center frequency $f_{\mathrm{IF}}$, the filtered product is down converted into in-phase $(I)$ and quadrature $(Q)$ components, and the time average sense cross correlation is obtained by averaging the summation of low-pass-filtered $I / Q$ detector outputs. To analyze the estimated cross correlation, we express the transmitted bandpass noise $x(t)$ using the mathematical narrowband random process model (McDonough and Whalen 1995):

$$
x(t)=x_{c}(t) \cos \left(2 \pi f_{0} t\right)-x_{s}(t) \sin \left(2 \pi f_{0} t\right),
$$

where $f_{0}$ is the carrier frequency and $x_{c}(t)$ and $x_{s}(t)$ are said to the in-phase and quadrature components of $x(t)$, respectively. Since $x_{c}(t)$ and $x_{s}(t)$ are independent lowpass random noise with Gaussian distribution with equal variances and zero means, $E\left[x_{c}(t)^{2}\right]=E\left[x_{s}(t)^{2}\right]$, $E\left[x_{c}(t)\right]=E\left[x_{s}(t)\right]=0$, and $E\left[x_{c}(t) x_{s}(t)\right]=0$.

The return signal from the $k$ th point scatterer can be modeled as

$$
x_{r k}(t)=x_{r c k} \cos \left(B_{1}\right)-x_{r s k} \sin \left(B_{1}\right),
$$

where $B_{1}=2 \pi f_{0} t-2 \pi f_{0} \tau_{r k}, \tau_{r k}$ is the return delay time corresponding to the $k$ th scatterer (depending on $t$ for a moving scatterer), and $x_{r c k}$ and $x_{r s k}$ are the scaled and delayed versions of $x_{c}(t)$ and $x_{s}(t)$, respectively: 


$$
\begin{aligned}
& x_{r c k}=\kappa_{r_{k}} x_{c}\left(t-\tau_{r k}\right), \\
& x_{r s k}=\kappa_{r_{k}} x_{s}\left(t-\tau_{r k}\right),
\end{aligned}
$$

where $\kappa_{r_{k}}$ represents the amplitude scaling factor contributed by the propagation path of the $k$ th scatterer.

When there exist multiple scatterers in the propagation path, the overall signal recovered by a receiving sensor is the superposition of the individual return signal from scatterers:

$$
X_{r}(t)=\sum_{k=1}^{K} x_{r k}(t)+n_{r}(t)
$$

where $K$ is the number of scatterers, $n_{r}(t)$ is the additive system noise in the return channel, and $x_{r k}(t)$ represents the return signal from the $k$ th scatterer.

The time-delayed and frequency-shifted replica of the transmitted signal can be modeled as

$$
x_{d}(t)=V_{c} \cos \left(B_{2}\right)-V_{s} \sin \left(B_{2}\right),
$$

where $B_{2}=2 \pi\left(f_{0}-f_{\mathrm{IF}}\right) t-2 \pi f_{0} \tau_{d}, f_{\mathrm{IF}}$ is the frequency offset referred to as the intermediate frequency (IF), and $V_{c}$ and $V_{s}$ are given by

$$
\begin{aligned}
& V_{c}=\kappa_{d} x_{c}\left(t-\tau_{d}\right)+n_{d c}(t)=x_{d c}(t)+n_{d c}(t) \\
& V_{s}=\kappa_{d} x_{s}\left(t-\tau_{d}\right)+n_{d s}(t)=x_{d s}(t)+n_{d s}(t),
\end{aligned}
$$

where $\tau_{d}$ is the delay time provided by the delay line, and $n_{d c}(t)$ and $n_{d s}(t)$ are the additive system noises in the delayed channel. Note that since the delayed signal is typically available at the receiver, these noise levels tend to be substantially lower than those for the return signals.

Assuming the transmitted noise signal $x(t)$ is a WSS ergodic and even symmetric process, we have $E\left[x_{r c k}(t) x_{d s}(t-\right.$ $\tau)] \approx E\left[x_{r s k}(t) x_{d c}(t-\tau)\right] \approx 0$. Consequently, the approximate time average sense $I$ and $Q$ components of cross correlation in the discrete domain (with $i$ denoting $t_{i}=i$ $\left.T_{\text {int }}\right)$ can be shown to be

$$
\begin{gathered}
\hat{R}_{I}=\frac{1}{N} \sum_{i=1}^{N} \sum_{k=1}^{K} x_{r c k_{i}} V_{c i} \cos \left(\theta_{k}\right)+n_{r c} V_{c i} \cos \left(\theta_{k}\right), \\
\hat{R}_{Q}=\frac{1}{N} \sum_{i=1}^{N} \sum_{k=1}^{K}-x_{r c k_{i}} V_{c i} \sin \left(\theta_{k}\right)-n_{r c} V_{c i} \sin \left(\theta_{k}\right),
\end{gathered}
$$

where $i$ is the noise sample index, $N=2 \beta T_{\text {int }}$ is the number of independent integrated noise samples, $T_{\mathrm{int}}$ is the measuring time, $\theta_{k}=2 \pi f_{0} \tau_{k}$ and $\tau_{k}=\tau_{d}-\tau_{r k}$ corresponds to the $k$ th return delay $\tau_{r k}$, and $n_{r c}$ is the additive noise in the return channel.

\section{c. Output signal-to-noise ratio}

The output signal-to-noise ratio $\mathrm{SNR}_{o}$ of a $\mathrm{CW}$ noise radar can be estimated by (Dawood 2001):

$$
\mathrm{SNR}_{o}=\frac{E^{2}\left[\hat{R}_{\mathrm{env}}\right]}{\operatorname{var}\left[\hat{R}_{\mathrm{env}}\right]},
$$

where $E[\cdot]$ represents the expectation operator, $\operatorname{var}[\cdot]$ denotes the variance operation, and $\hat{R}_{\text {env }}$ is the envelope of the estimated cross correlation.

When the number of independent integrated noise samples is large (i.e., $N \gg 1$ ), unbiased cross-correlation estimates are obtained, hence $E^{2}\left[\hat{R}_{\text {env }}\right]$ can be shown to be

$$
E^{2}\left[\hat{R}_{\mathrm{env}}\right]=E^{2}\left[\hat{R}_{I}\right]+E^{2}\left[\hat{R}_{Q}\right] .
$$

From Eqs. (11) and (12), $E^{2}\left[\hat{R}_{\text {env }}\right]$ is approximated as

$$
E^{2}\left[\hat{R}_{\mathrm{env}}\right]=\sum_{k=1}^{K} R_{c c_{k}}^{2}+\sum_{k=1}^{K} \sum_{k \neq m=1}^{K} R_{c c_{m}} R_{c c_{k}} \cos \left(\theta_{m}-\theta_{k}\right),
$$

where $k$ and $m$ are the scatterer indices, $\theta_{k}=2 \pi f_{0} \tau_{k}$, $\theta_{m}=2 \pi f_{0} \tau_{m}, \tau_{k}=\tau_{d}-\tau_{r k}$, and $\tau_{m}=\tau_{d}-\tau_{r m}$. Straightforwardly, $E^{2}\left[\hat{R}_{\text {env }}\right]$ can be rewritten as

$$
E^{2}\left[\hat{R}_{\mathrm{env}}\right]=\left[\sum_{k=1}^{K} R_{c c_{k}} \cos \left(\theta_{k}\right)\right]^{2}+\left[\sum_{k=1}^{K} R_{c c_{k}} \sin \left(\theta_{k}\right)\right]^{2},
$$

where $R_{c c_{k}}$ is the correlation corresponding to the $k$ th return signal, as defined by

$$
R_{c c_{k, m}}=E\left[x_{r c_{k}} x_{d c}\right]=E\left[x_{r s_{k}} x_{d s}\right],
$$

where $x_{r c_{k}}=x_{c}\left(t-\tau_{r k}\right), x_{r s_{k}}=x_{s}\left(t-\tau_{r k}\right), x_{d c}=x_{c}\left(t-\tau_{d}\right)$ and $x_{d s} \stackrel{{ }_{k}}{=} x_{s}\left(t-\tau_{d}\right)$. Both $x_{c}(t)$ and $x_{s}(t)$ are low-pass random noise with uniform PSD, so we have $R_{c c_{k}}=$ $\sin c\left[\beta\left(\tau_{d}-\tau_{r k}\right)\right]$. Inserting Eqs. (11) and (12) in the following equation, $E\left[\hat{R}_{\text {env }}^{2}\right]=E\left[\hat{R}_{I}^{2}\right]+E\left[\hat{R}_{Q}^{2}\right], E\left[\hat{R}_{\text {env }}^{2}\right]$ can be shown to be

$$
\begin{array}{r}
E\left[\hat{R}_{\mathrm{env}}^{2}\right]=\frac{1}{N}\left(\sum_{k=1}^{K} S_{r k} S_{d}+\sum_{k=1}^{K} S_{r k} N_{d}+N_{r} S_{d}\right. \\
\left.+N_{r} N_{d}+(N+1) E^{2}\left[\hat{R}_{\mathrm{env}}\right]\right),
\end{array}
$$

where $N=2 \beta T_{\text {int }}$ is the number of independent integrated noise samples (i.e., the time-bandwidth product); $T_{\text {int }}$ represents the measuring time, $E^{2}\left[\hat{R}_{\text {env }}\right]$ is given by Eq. (15); $S_{r k}$ and $S_{d}$ represent the signal power of the $k$ th return signal and the delayed replica, respectively; and $N_{r}$ and $N_{d}$ are the additive noise power in the return and delayed channels, respectively. Subtracting $E^{2}\left[\hat{R}_{\text {env }}\right]$ 
given by Eq. (15) from $E\left[\hat{R}_{\text {env }}^{2}\right]$ given by Eq. (18), $\operatorname{var}\left[\hat{R}_{\text {env }}\right]$ can be expressed by

$$
\begin{aligned}
\operatorname{var}\left[\hat{R}_{\mathrm{env}}\right]=\frac{1}{N}( & \sum_{k=1}^{K} S_{r k} S_{d}+\sum_{k=1}^{K} S_{r k} N_{d} \\
& \left.+N_{r} S_{d}+N_{r} N_{d}+E^{2}\left[\hat{R}_{\mathrm{env}}\right]\right)
\end{aligned}
$$

Consequently, the approximate output signal-to-noise ratio, $\widehat{\mathrm{SNR}}_{o}$, at the correlator defined as

$$
\widehat{\mathrm{SNR}}_{o} \equiv \frac{E^{2}\left[\hat{R}_{\mathrm{env}}\right]}{\operatorname{var}\left[\hat{R}_{\mathrm{env}}\right]}
$$

can be written as

$$
\widehat{\mathrm{SNR}}_{o}=\frac{T_{\mathrm{int}} E^{2}\left[\hat{R}_{\mathrm{env}}\right]}{\frac{1}{2 \beta}\left(\sum_{k=1}^{K} S_{r k} S_{d}+\sum_{k=1}^{K} S_{r k} N_{d}+N_{r} S_{d}+N_{r} N_{d}+E^{2}\left[\hat{R}_{\mathrm{env}}\right]\right)} .
$$

\section{d. Cross-correlation model}

Based on the approximate $\widehat{\mathrm{SNR}}_{o}$ given by Eq. (21), we can model the estimated cross correlation as a noisy signal $R_{M}$ :

$$
R_{M}(t)=S_{M}(t)+n_{M}(t)
$$

where $S_{M}(t)$ represents the true signal with envelope $\sqrt{E^{2}\left[\hat{R}_{\text {env }}\right]}$ and energy $T_{\text {int }} E^{2}\left[\hat{R}_{\text {env }}\right]$ in measuring time $T_{\text {int }}$, and $n_{M}(t)$ is the complex additive Gaussian noise component at the output of the correlator. The large number of random contributions summed in the coherent integration leads to the assertion of normality in the simplified model. In addition, at this point the computational savings of the model are clear-the generation of one sample per integration time, $T_{\text {int }}$, versus the generation operations and filtering at the Nyquist rate required by the signal bandwidth gives at least a factor of $N$ reduction per observation. This reduction is even greater when subsequent filtering and other operations are included in the assessment and even more when the simulation signals are sampled at a higher than Nyquist rate as is done in the present work.

From Eq. (16), the estimated cross correlation can be generally modeled as a complex signal $R_{M}=R_{I M}-$ $j R_{Q M}$ with the following modeled $I$ and $Q$ components:

$$
\begin{aligned}
R_{I M} & =\sum_{k=1}^{K} A_{r k} \operatorname{sinc}\left(\beta \tau_{k}\right) \cos \left(\theta_{k}\right)+n_{I}(t), \\
R_{Q M} & =\sum_{k=1}^{K} A_{r k} \operatorname{sinc}\left(\beta \tau_{k}\right) \sin \left(\theta_{k}\right)+n_{Q}(t),
\end{aligned}
$$

where $K$ is the number of scatterers, $\theta_{k}=2 \pi f_{0} \tau_{k}, \tau_{k}=$ $\tau_{d}-\tau_{r k}, \tau_{r k}$ represents the return delay corresponding to the $k$ th scatterer, $A_{r k}$ represents the amplitude scaling factor determined by the return signal power $S_{r k}$ due to the propagation path and the integration time $T_{\mathrm{int}}$, and $n_{I}(t)$ and $N_{q}(t)$ are independent Gaussian random noise samples added to provide the modeled output signal-to-noise ratio, $\widehat{\mathrm{SNR}}_{o}$.

\section{e. Illustration of cross-correlation model}

Figure 1 depicts an illustration of the cross-correlation model and actual estimated cross-correlation verification process for multiple backscattering. Simulations are performed to verify this cross-correlation model in conjunction with a time-domain simulation of the estimated cross correlation.

Figures 2 and 3 show the envelopes of the continuous time-domain-estimated cross correlations and the computationally simpler modeled cross correlations when 100 scatterers are randomly situated within one range bin with the range center of 37.5 and $375 \mathrm{~m}$, respectively. By time-domain simulations we are referring to sampling at 10 times the simulation bandwidth and performing all time-domain operations required (i.e., correlation and filtering). Hence the computational savings over the time-domain approach are on the order of $10000: 1$ using the simplified model. It can be seen from the figures that the computationally simple modeled cross-correlation envelopes are consistent with the estimated cross-correlation envelopes using the timedomain simulation.

Figures 4 and 5 show the PSDs of the modeled and time-domain-estimated cross correlations, respectively. In this simulation, 100 scatterers are initialized with 


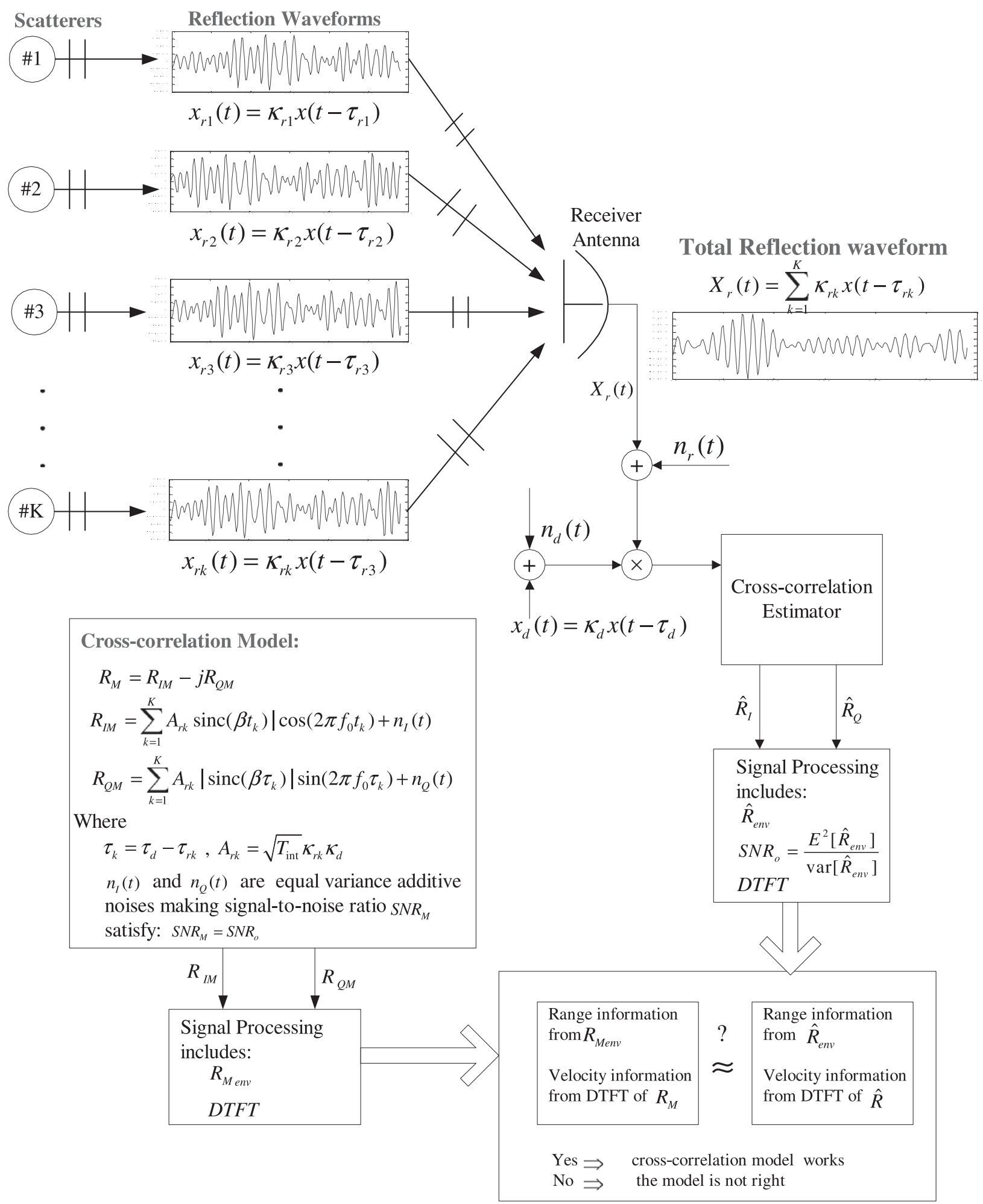

FIG. 1. Illustration of the modeled and estimated cross-correlation verification process. Here $\tau_{r k}$ is the return delay corresponding to the $k$ th scatterer, $\tau_{k}=\tau_{d}-\tau_{k}$. $\mathrm{SNR}_{M}=\mathrm{SNR}_{o}$. 


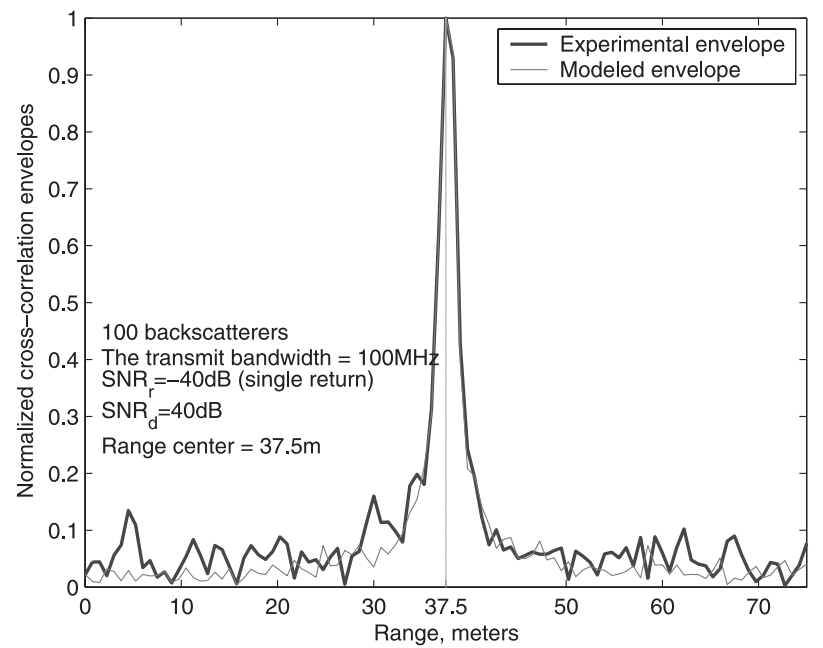

FIG. 2. Envelopes of the estimated and modeled cross correlations. Input signal-to-noise ratios in the return and delayed channels are set as $\mathrm{SNR}_{r}=-40 \mathrm{~dB}$ and $\mathrm{SNR}_{d}=40 \mathrm{~dB}$, respectively. The transmit bandwidth $\beta=100 \mathrm{MHz}$.

randomly assigned positions within a desired range bin and with randomly assigned velocities ranging from 4.7 to $5.3 \mathrm{~m} \mathrm{~s}^{-1}$ with mean $5 \mathrm{~m} \mathrm{~s}^{-1}$ and standard derivation $0.1 \mathrm{~m} \mathrm{~s}^{-1}$. From these figures it is shown that the extracted velocity information from the cross-correlation model is consistent with that from the estimated cross correlation using the time-domain simulation, and the estimated velocities based on the estimated and modeled cross correlation are located in the velocity range from 4.7 to $5.3 \mathrm{~m} \mathrm{~s}^{-1}$.

Based on above analysis and simulation results, we can confirm that the computationally efficient modeled

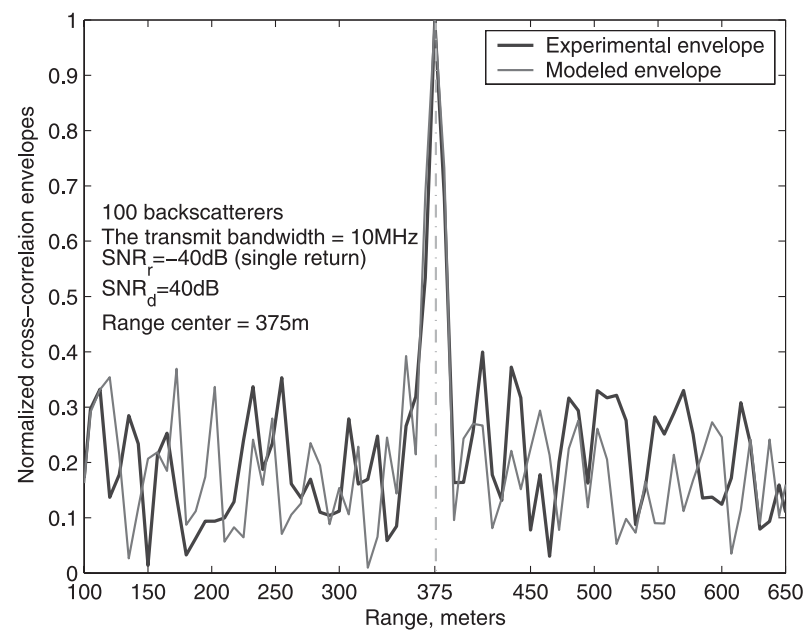

FIG. 3. Envelopes of simulated and modeled cross correlation. Input signal-to-noise ratios in the return and delayed channels are set as $\mathrm{SNR}_{r}=-40 \mathrm{~dB}$ and $\mathrm{SNR}_{d}=40 \mathrm{~dB}$, respectively. The transmit bandwidth $\beta=10 \mathrm{MHz}$.

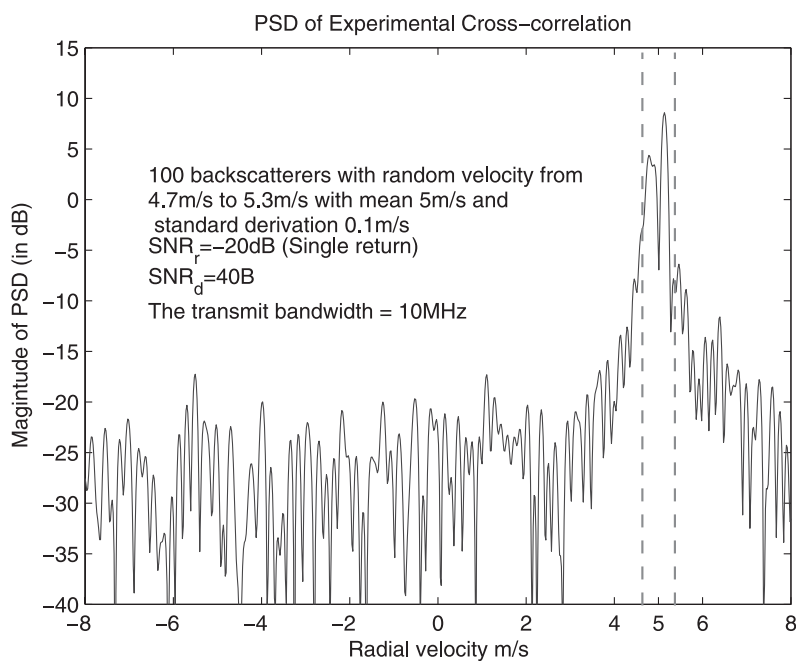

FIG. 4. The PSD of the estimated cross correlation. The transmit bandwidth $\beta=10 \mathrm{MHz}$, and input signal-to-noise ratios in the return and delayed channels are set as $\mathrm{SNR}_{r}=-20 \mathrm{~dB}$ and $\mathrm{SNR}_{d}=40 \mathrm{~dB}$, respectively.

cross correlation described by Eqs. (23) and (24) can be used to simulate the actual estimated cross correlation derived from a time-domain simulation for range and velocity estimation.

\section{Atmospheric backscattering CRI simulation results}

In this section, a modification of an existing atmospheric backscattering model is presented for the

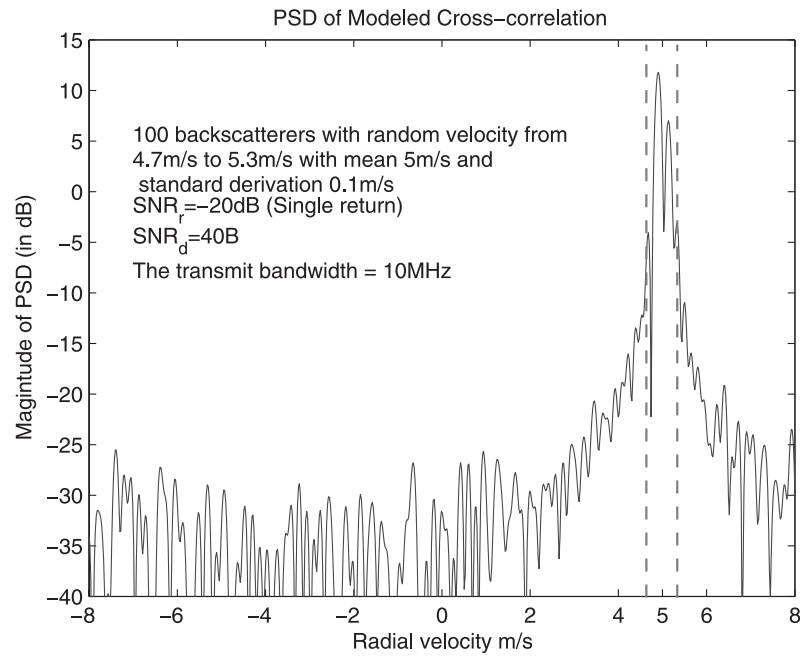

FIG. 5. The PSD of the modeled cross-correlation results. The transmit bandwidth $\beta=10 \mathrm{MHz}$, and input signal-to-noise ratios in the return and delayed channels are set as $\mathrm{SNR}_{r}=-20 \mathrm{~dB}$ and $\mathrm{SNR}_{d}=40 \mathrm{~dB}$, respectively. 


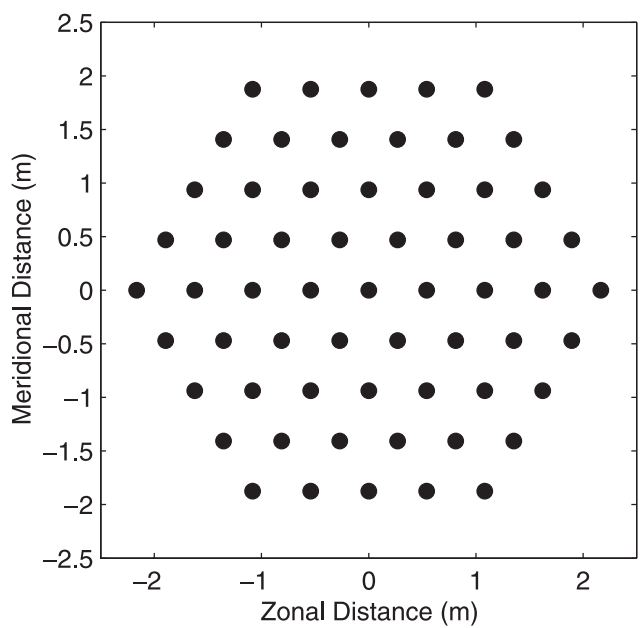

FIG. 6. Geometry of the TEP array with 61 sensors arranged in a hexagonal lattice. The distance between any two neighbor sensors is approximately $0.5412 \mathrm{~m}$ (Cheong et al. 2004b).

atmospheric backscattering simulation when a coherent CW noise radar is applied. The existing model used in the present work was originally developed by Holdsworth and Reid (1995) for both SA and pulsed radar simulations. In its implementation for pulsed radar CRI, some more realistic modifications were proposed by $\mathrm{Yu}$ (2000), and a more efficient simulation algorithm of turbulent wind field updates was proposed by Cheong et al. (2004a) to reduce the computational load incurred with very large numbers of scatterers.

In the simulation model of Holdsworth and Reid (1995) a large number of scatterers in a 3D enclosing volume is used to simulate bulk atmospheric backscattering. The scatterers are initialized with random reflectivities and with randomly assigned locations. The total received signal is simulated as a superposition of individual complex signals corresponding to reflections from scatterers in that enclosing volume. As was shown in the previous section, for a given set of scatterers, the computationally efficient modeled correlation at the receiver output is approximately equal to correlation estimated by the computationally intensive, time-domain simulation of CW noise radar signals. Hence, for a CRI backscattering simulation (Cheong et al. 2004a), this model can be used for the received $\mathrm{CW}$ correlations from a set of scatterers at each of the receivers. Since CRI typically requires a large number of receivers, an efficient means of computing the $\mathrm{CW}$ noise radar returns from the set of scatterers is key to feasible simulation of these systems. The modeled correlations for each receiver for a given SNR are generated using the amplitude scaling and phases for each of the scatterers used in the model in accord with the terms in Eqs. (23) and (24).
TABLE 1. Simulation parameters are based on the turbulent eddy profiler specifications. [Adapted from Mead et al. (1998).]

\begin{tabular}{ll}
\hline \hline Center frequency & $f_{0}=915 \mathrm{MHz}$ \\
Receiver array & 61 elements \\
One range gate with center & $950 \mathrm{~m}$ \\
Demodulated signal sampling rate & $140 \mathrm{~Hz}$ \\
Transmitter pointing direction & Vertical \\
No. of scatterers & 10000 \\
Horizontal wind magnitude & $25 \mathrm{~m} \mathrm{~s}^{-1}$ \\
Vertical wind & $0 \mathrm{~m} \mathrm{~s}^{-1}$ \\
Azimuth angle & $45^{\circ}$
\end{tabular}

CRI for coherent CW noise radar was tested by processing the modeled signals using the atmospheric backscattering model discussed in section 2 . The simulation results including the echo power estimates, the radial velocity estimates, and $3 \mathrm{D}$ wind field estimates are demonstrated. To verify the simulation results using coherent $\mathrm{CW}$ noise radar, widely accepted simulation results using pulsed radar under identical simulation conditions are comparatively shown.

\section{a. Simulation radar specifications}

For the purpose of comparison, the receiver array of the simulated coherent $\mathrm{CW}$ noise radar is assumed to have the same sensor configuration as that of the simulated turbulent eddy profiler (TEP) array by Cheong et al. (2004b), as shown in Fig. 6. This configuration has 61 sensors arranged in a hexagonal lattice to mimic the TEP radar developed at the University of Massachusetts, Amherst (Mead et al. 1998; Pollard et al. 2000; Dekker and Frasier 2004).

In the simulations of CRI using the TEP performed by Cheong et al. (2004b) the range resolution is $\Delta r=33.3 \mathrm{~m}$ corresponding to a transmitted pulse width of $\tau_{p}=222 \mathrm{~ns}$ in the TEP radar. To get an equivalent range resolution of $33.3 \mathrm{~m}$ in the simulation of CRI using coherent $\mathrm{CW}$ noise radar, the transmit bandwidth is set as $\beta=4.5455 \mathrm{MHz}$. Based on the TEP radar specifications listed by Cheong et al. (2004b), the basic radar specifications used in the following simulations are listed in Table 1. Note that there are implementation differences between pulsed and CW radars. Typically, CW radars have lower peak power and much higher duty cycles than pulsed radarsthese parameters can be varied to impact the return SNR from radar systems. For the purpose of side-by-side imaging comparison, we have assumed the returned postcorrelation SNRs from the two systems are identical.

\section{b. Simulation results}

In the first simulation, the atmospheric reflectivity is simulated as a single Gaussian blob centered at $\left(0^{\circ}, 0^{\circ}\right)$ with $\sigma_{x}=0^{\circ}, \sigma_{x}=0^{\circ}, \rho=0.0$. Given the mean wind field 


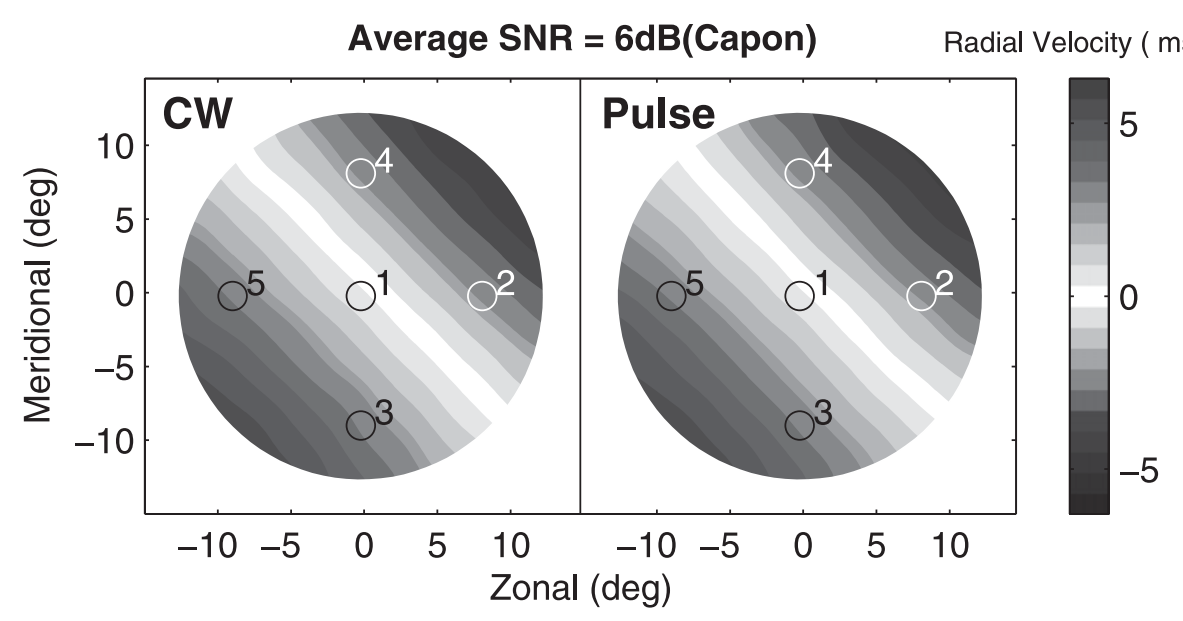

\section{Doppler Spectrum}

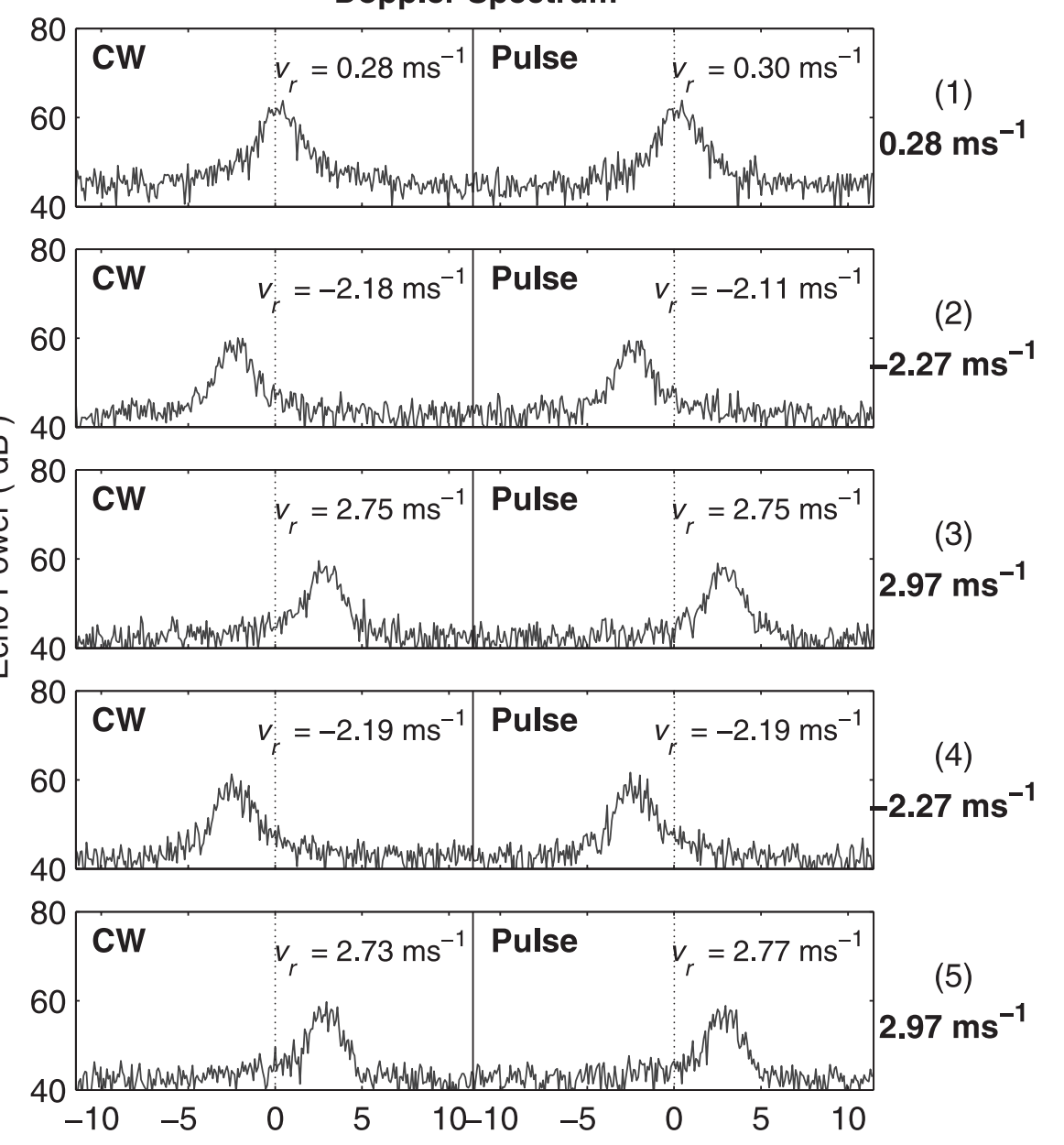

FIG. 7. Radial velocity is estimated using the Capon PPB method and the Doppler spectra of five selected pixels is estimated using the periodogram technique. The true velocities for five pixels are stated to the right in bold. The reflectivity model is a single Gaussian blob centered at $\left(0^{\circ}, 0^{\circ}\right)$ with $\sigma_{x}=0^{\circ}$, $\sigma_{x}=0^{\circ}$, and $\rho=0.0$. 

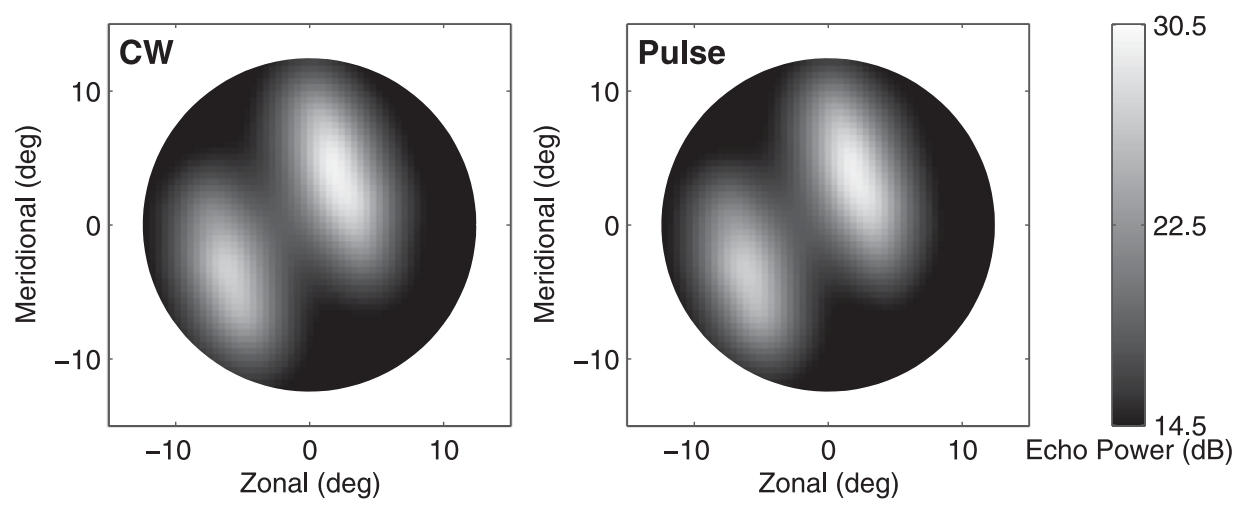

(b) Estimated Radial Velocities
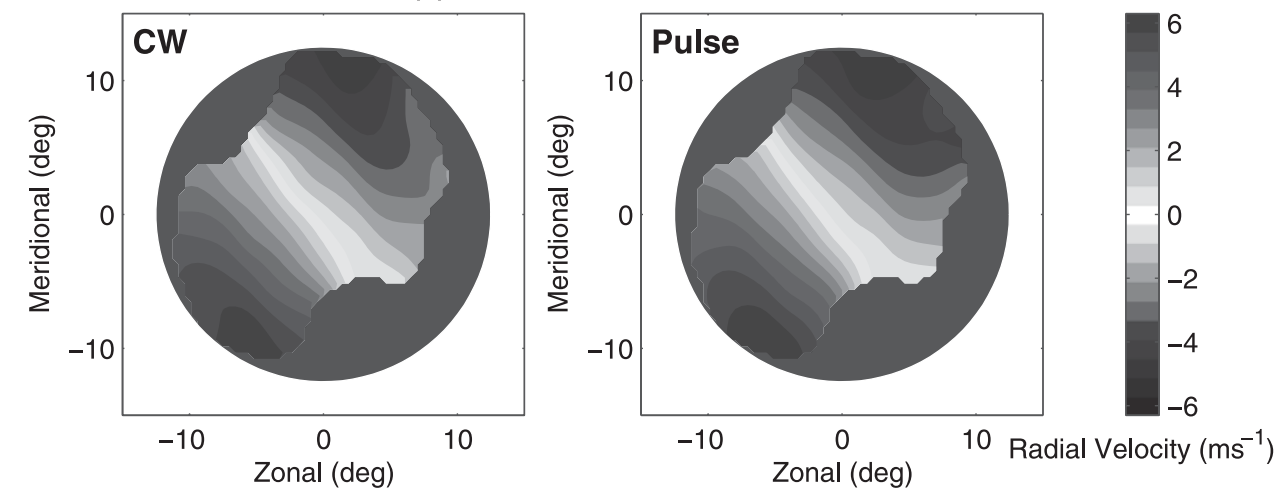

(c) Estimated 3-D Wind Field Maps
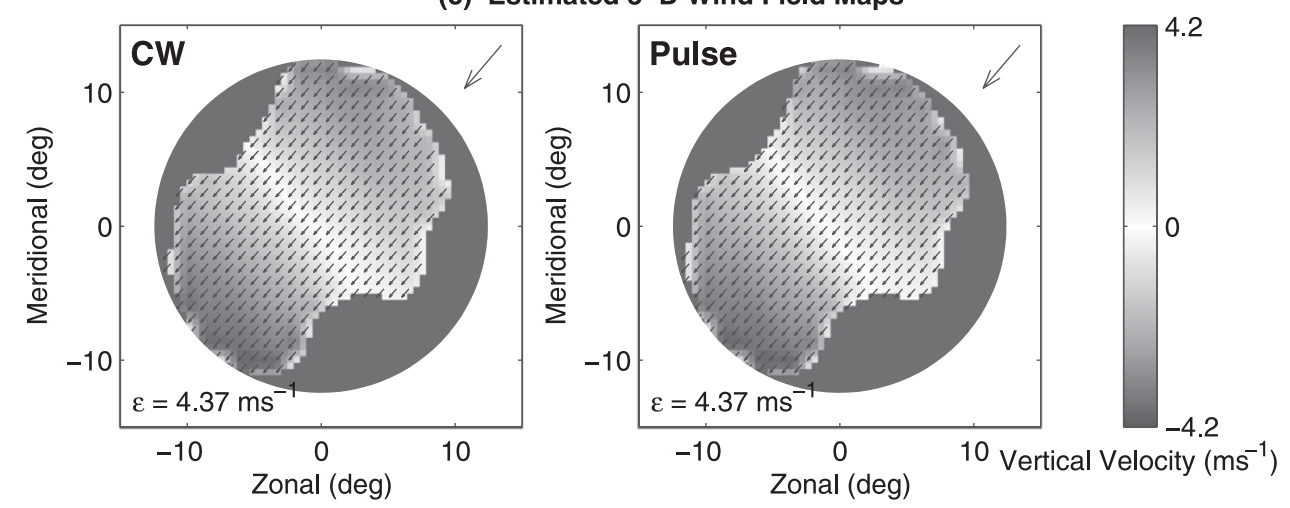

FIG. 8. Estimated echo power, radial velocity, and wind field maps. Echo power and radial velocity are estimated using the Capon PPB method. Radial velocity maps are shown for the region with the SNR > $3 \mathrm{~dB}$. Turbulent velocity RMS $=0 \mathrm{~m} \mathrm{~s}^{-1}$.

listed in Table 1 with zero turbulent velocity, the radial velocity estimates are obtained using the Capon pulsepair beamforming (PPB) method (Cheong et al. 2004b) and the periodogram technique, separately. Figure 7 shows the radial velocity contour lines obtained using the Capon PPB method and the Doppler spectra of five distinct pointing directions using the periodogram technique. For both the $\mathrm{CW}$ noise radar and the pulsed radar, the expected negative and positive radial velocities are found in the upper-right and lower-left regions of the two top panels, respectively, the radial velocities for five selected pointing directions listed in their corresponding Doppler spectra are very close to the true radial velocities, and the Doppler spectra are consistent with the respective radial velocity maps.

In the second simulation, the echo powers, radial velocities, and 3D wind fields for a random reflectivity model with two Gaussian blobs are estimated. The simulated reflectivity model is a sum of two Gaussian blobs centered at $\left(2^{\circ}, 4^{\circ}\right)$ with $\sigma_{x}=2^{\circ}, \sigma_{x}=2^{\circ}, \rho=-0.6$, 

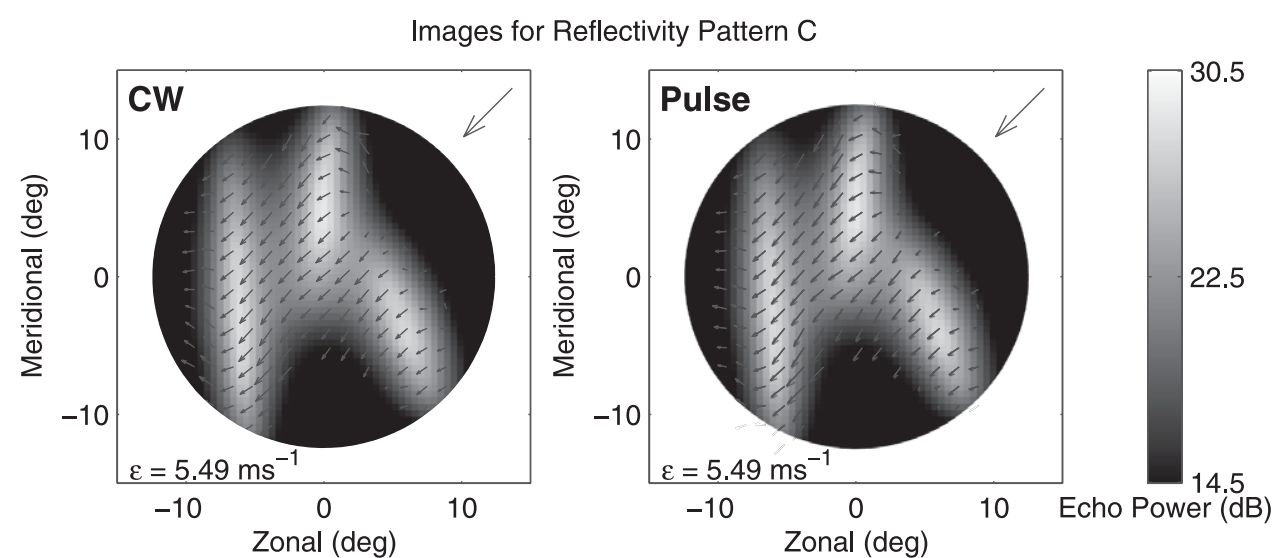

Images for Reflectivity Pattern D
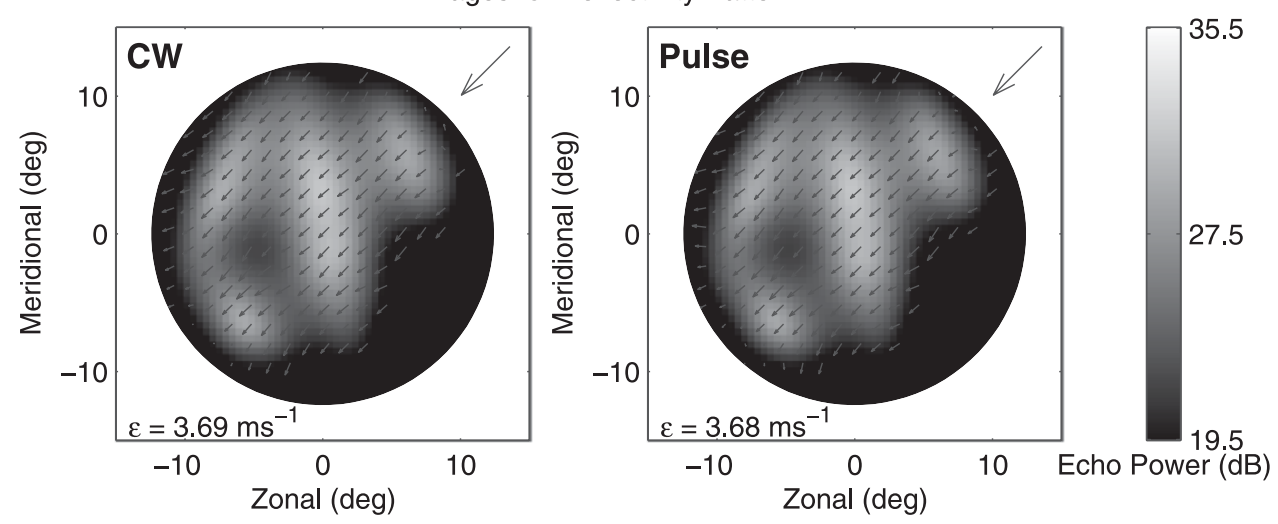

FIG. 9. The 2D horizontal wind field estimates superimposed over echo power estimates. Turbulence velocity $\mathrm{RMS}=0 \mathrm{~m} \mathrm{~s}^{-1}$.

and at $\left(-4^{\circ}, 6^{\circ}\right)$ with $\sigma_{x}=-4^{\circ}, \sigma_{x}=-6^{\circ}, \rho=-0.6$, respectively. A constant northeasterly horizontal wind of $25 \mathrm{~m} \mathrm{~s}^{-1}$ with no vertical velocity and no turbulent velocity is used in this simulation. Figure 8 shows the corresponding simulation results. As shown in Fig. 8a, both set of estimated echo powers are consistent with the reflectivity model. From the estimated radial velocity contour lines, shown in Fig. 8b, two expected radial velocities of -6.45 and $+6.45 \mathrm{~m} \mathrm{~s}^{-1}$ can be observed at the edge of the circle $\left(12.5^{\circ}\right)$ at northeast (top right) and southwest (bottom left), respectively, for both panels. Figure $8 \mathrm{c}$ has indicated two similar 3D wind field estimates and corresponding RMS errors. In Fig. 8c, the true horizontal wind vector is indicated by a single arrow in the upper-right corner of each image for reference and the RMS error of the estimated wind fields is provided in the bottom-left corner. The uncannily close similarity in the RMS errors is explained by the fact that both simulations are using identical scatterer distributions for comparison purposes.

The final two simulations are performed to observe the effects of reflectivity variations on wind field esti- mates. The mean wind field is set up to be uniform horizontal wind of $25 \mathrm{~m} \mathrm{~s}^{-1}$ from $45^{\circ}$ azimuth with no vertical velocity. Figures 9 and 10 show the $2 \mathrm{D}$ wind field estimates superimposed on echo power estimates for two reflectivity models when no turbulent field or a turbulent wind field with an RMS of $\pm 1 \mathrm{~m} \mathrm{~s}^{-1}$ was added on the top of the mean wind field, respectively. From Figs. 9 and 10, similar echo power estimates and wind field estimates obtained by using both $\mathrm{CW}$ noise radar and pulsed radar are observed, and the estimated 2D wind field and corresponding RMS errors shown in the lower-left corner of each panel have indicated that the impact of reflectivity variations on wind field estimates obtained using CW noise radar and pulsed radar are same for the mean field with and without turbulent field.

\section{Conclusions and discussion}

A simulation capability for CRI using a spaced antenna system receiving $\mathrm{CW}$ noise radar returns has been described and verified. Potential uses of this simulation 

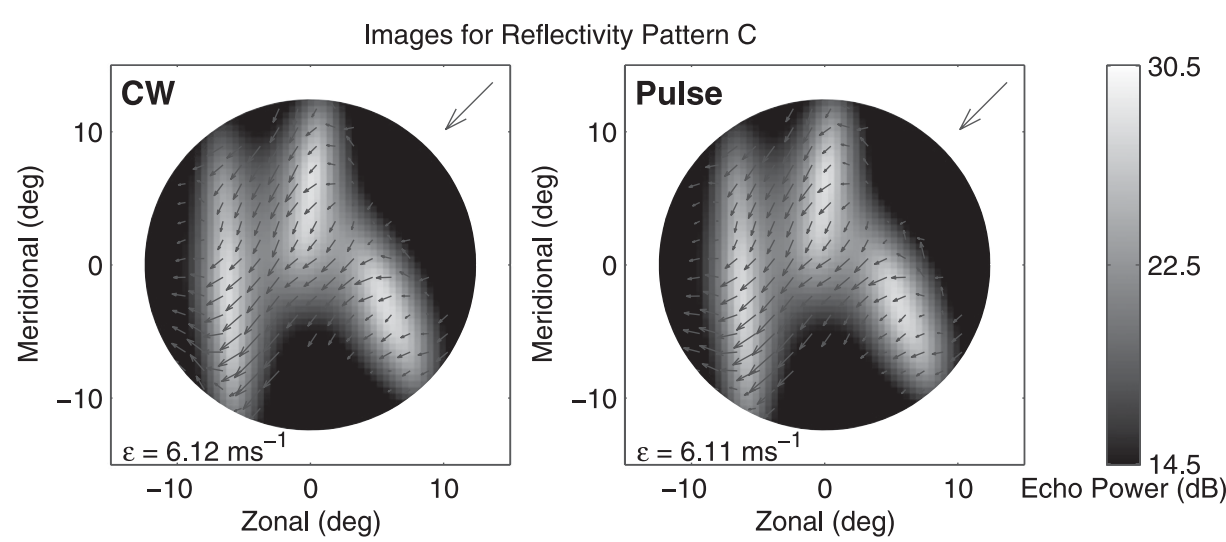

Images for Reflectivity Pattern D
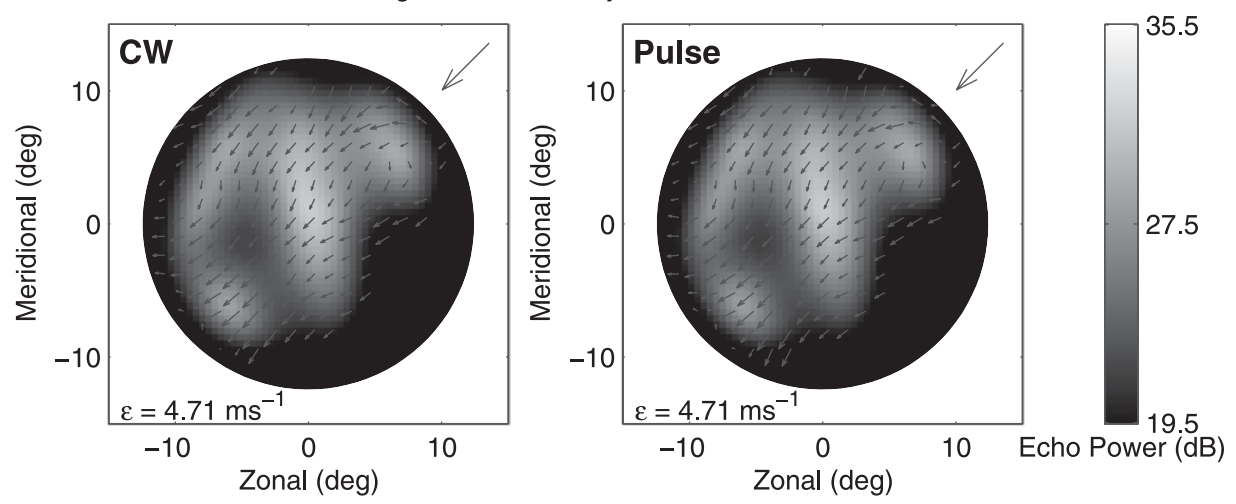

FIG. 10. The 2D horizontal wind field estimates superimposed over estimated reflectivity. A turbulent wind field with RMS of $\pm 1 \mathrm{~m} \mathrm{~s}^{-1}$ was added on the mean wind field, which is indicated by an arrow in the upper-right corner of each panel.

include passive noise radar development for CRI using existing communication signals. A computationally efficient cross-correlation model for coherent CW noise radar from multiple scatterers has been developed and verified via comparison with a computationally intensive time-domain simulation. Based on the cross-correlation model, modifications have been made to an existing atmospheric scattering model previously used for spaced antenna pulsed radar simulations. Given the same simulation conditions, similar CRI simulation results for CW noise and pulsed radar are observed by using the efficient model. From this effort we draw two conclusions. First, accurate and efficient simulation of the coherent $\mathrm{CW}$ noise radars for atmospheric CRI is possible using the approach presented in this paper. Second, based on the initial side-by-side comparisons of a CW noise radar array and a pulsed radar array, it appears that CW noise CRI of the atmosphere is a promising technique that warrants further study-particularly interesting is the exploitation of extant communication signals for atmospheric imaging.

\section{REFERENCES}

Cheong, B. L., M. W. Hoffman, and R. D. Palmer, 2004a: Efficient atmospheric simulation for high resolution radar imaging applications. J. Atmos. Oceanic Technol., 21, 374-378.

,,--- S. J. Frasier, and F. J. Lopez-Dekker, 2004b: Pulse pair beamforming and the effects of reflectivity field variations on imaging radars. Radio Sci., 39, RS3014, doi:10.1029/2002RS002843.

Dawood, M., 2001: Ultrawideband coherent random noise radar theory and experiments. Ph.D. thesis, University of Nebraska at Lincoln, 168 pp.

Dekker, P. L., and S. J. Frasier, 2004: Radio acoustic sounding with a UHF volume imaging radar. J. Atmos. Oceanic Technol., 21, 766-776.

Doviak, R. J., and D. S. Zrnić, 1993: Doppler Radar and Weather Observations. 2nd ed. Academic Press, 562 pp.

Guosui, L., G. Hong, and S. Weimin, 1999: Development of random signal radars. IEEE Trans. Aerosp. Electron. Syst., 35, 770-777.

Holdsworth, D. A., and I. M. Reid, 1995: A simple model of atmospheric radar backscatter: Description and application to the full correlation analysis of spaced antenna data. Radio Sci., 30, 1263-1280.

Hysell, D. L., 1996: Radar imaging of equatorial $F$ region irregularities with maximum entropy interferometry. Radio Sci., 31, 1567-1578. 
Krehbiel, P. R., and M. Brook, 1979: A broadband noise technique for fast scanning radar observations of clouds and clutter targets. IEEE Trans. Geosci. Electron., GE-17, 196-204.

Kudeki, E., and F. Sürücü, 1991: Radar interferometric imaging of field-aligned plasma irregularities in the equatorial electrojet. Geophys. Res. Lett., 18, 41-44.

McDonough, R. M., and A. Whalen, 1995: Detection of Signals in Noise. 2nd ed. Academic Press, 495 pp.

Mead, J. B., G. Hopcraft, S. J. Frasier, B. D. Pollard, C. D. Cherry, D. H. Schaubert, and R. E. McIntosh, 1998: A volumeimaging radar wind profiler for atmospheric boundary layer turbulence studies. J. Atmos. Oceanic Technol., 15, 849-859.

Meyer, M. G., and J. D. Sahr, 2004: Passive VHF radar interferometer implementation and observations. Radio Sci., 39, RS3008, doi:10.1029/2003RS002985.

Narayanan, R. M., Y. Xu, P. D. Hoffmeyer, and J. O. Curtis, 1998: Design, performance, and implementation of a coherent ultrawideband random noise radar. Opt. Eng., 37, $1855-1869$

Pollard, B. D., S. Khanna, S. J. Frasier, J. C. Wyngaard, D. W. Thomson, and R. E. McIntosh, 2000: Local structure of the convective boundary layer from a volume-imaging radar. J. Atmos. Sci., 57, 2281-2296.
Reid, M. S., 1969: A millimeter wave pseudorandom coded meteorological radar. IEEE Trans. Geosci. Electron., GE-7, 146-156.

Sahr, J. D., and F. D. Lind, 1997: The Manastash Ridge radar: A passive bistatic radar for upper atmospheric radio science. Radio Sci., 32, 2345-2358.

—, and M. Meyer, 2004: Opportunities for passive VHF radar studies of plasma irregularities in the equatorial $\mathrm{E}$ and $\mathrm{F}$ regions. J. Atmos. Sol.-Terr. Phys., 66, 1675-1681.

Waltman, W., C. D. McGillem, and G. R. Cooper, 1966: A broadband interferometer. IRE Int. Convention Record, 14 (Part 5), 156-166.

Yanovsky, F. J., 2002: Potential of a Noise Radar for Meteorological Applications. Vols. 18-20, Proceedings of the First International Workshop on the Noise Radar Technology, 195-200.

Yu, T.-Y., 2000: Radar studies of the atmosphere using spatial and frequency diversity. Ph.D. thesis, University of NebraskaLincoln, $227 \mathrm{pp}$.

, R. D. Palmer, and P. B. Chilson, 2001: An investigation of scattering mechanisms and dynamics in PMSE using coherent radar imaging. J. Atmos. Sol.-Terr. Phys., 63, 1797-1810.

Zhang, Z., Z. Cao, and T. Zhang, 1999: Analysis and simulation of land and rain clutter for PRC CW radar at X-band. IEE Proc. Radar Sonar Navig., 146 (2), 101-106. 